\title{
DIFFERENTIAL OPERATORS AND FLAT CONNECTIONS ON A RIEMANN SURFACE
}

\author{
INDRANIL BISWAS
}

Received 21 December 2002

\begin{abstract}
We consider filtered holomorphic vector bundles on a compact Riemann surface $X$ equipped with a holomorphic connection satisfying a certain transversality condition with respect to the filtration. If $Q$ is a stable vector bundle of rank $r$ and degree $(1-$ genus $(X)) n r$, then any holomorphic connection on the jet bundle $J^{n}(Q)$ satisfies this transversality condition for the natural filtration of $J^{n}(Q)$ defined by projections to lower-order jets. The vector bundle $J^{n}(Q)$ admits holomorphic connection. The main result is the construction of a bijective correspondence between the space of all equivalence classes of holomorphic vector bundles on $X$ with a filtration of length $n$ together with a holomorphic connection satisfying the transversality condition and the space of all isomorphism classes of holomorphic differential operators of order $n$ whose symbol is the identity map.
\end{abstract}

2000 Mathematics Subject Classification: 32C38, 31A35, 14F10.

1. Introduction. Let $E$ be a holomorphic vector bundle of rank $(n+1) r$ over a compact connected Riemann surface $X$ equipped with a flat connection $\nabla$. Let $F$ be a holomorphic subbundle of $E$ of rank $r$. The second fundamental form of $\nabla$ gives a filtration of $V$

$$
F_{0}:=0 \subset F_{1}:=F \subset F_{2} \subset F_{3} \subset \cdots \subset F_{m}
$$

by subbundles. More precisely, $F_{i+1} / F_{i}$ is the subbundle of the vector bundle $E / F_{i}$ generated by the image of the second fundamental form

$$
S\left(F_{i}\right): T X \otimes F_{i} \longrightarrow E / F_{i}
$$

of the subbundle $F_{i}$ for the connection $\nabla$. This condition defines the filtration inductively. Let

$$
S_{i}: F_{i} / F_{i-1} \longrightarrow K_{X} \otimes\left(F_{i+1} / F_{i}\right)
$$

be the homomorphism induced by $S\left(F_{i}\right)$. We call the pair $(\nabla, F)$ a coupled connection if $m=n+1$ with $F_{n+1}=E$, and $S_{i}$ is an isomorphism for all $i \in$ $[1, n]$. 
Let $Q:=E / F_{n}$ be the final quotient in the above filtration for the coupled connection $(\nabla, F)$. We show that there is a natural isomorphism of $E$ with the jet bundle $J^{n}(Q)$ (Lemma 3.2). Using Lemma 3.2, we construct a differential operator

$$
D \in H^{0}\left(X, \operatorname{Diff}_{X}^{n+1}\left(Q, K_{X}^{n+1} \otimes Q\right)\right)
$$

of order $n+1$, where $K_{X}$ is the holomorphic cotangent bundle of $X$ such that the symbol of $D$ is the identity automorphism of $Q$.

Conversely, given such a differential operator whose symbol is the identity automorphism of $Q$, we construct a coupled connection on $J^{n}(Q)$ (Lemma 4.1).

Two coupled connections $(\nabla, F)$ on $E$ and $\left(\nabla^{\prime}, F^{\prime}\right)$ on $E^{\prime}$ are called equivalent if there is an isomorphism of $E$ with $E^{\prime}$ that takes $F$ to $F^{\prime}$ and $\nabla$ to $\nabla^{\prime}$. Similarly, two differential operators $D$ and $D^{\prime}$, on $Q$ and $Q^{\prime}$, respectively, of the above type with identity maps as symbol will be called equivalent if there is an isomorphism of $Q$ with $Q^{\prime}$ that transports $D$ to $D^{\prime}$.

We prove that the space of all equivalence classes of coupled connections is in bijective correspondence with the space of all equivalence classes of differential operators. More precisely, both of the above-mentioned constructions, namely, from operators to coupled connections and from coupled connections to operators, give a bijective correspondence and they are inverses of each other (Theorem 4.5).

Let $Q$ be a stable vector bundle over $X$ of rank $r$ and degree $(1-g) n r$, where $g$ is the genus of $X$. We prove that $J^{n}(Q)$ admits a flat connection (Lemma 3.6). It is also shown that any flat connection on $J^{n}(Q)$, together with the subbundle $K_{X}^{\otimes n} \otimes Q$, defines a coupled connection (Lemma 3.5).

The special case of $n=1$ was investigated in [2]. More on coupled connections can be found in [3].

2. Preliminaries. We recall the definition of jet bundles and its basic properties.

Let $E$ be a holomorphic vector bundle over a Riemann surface $X$ and let $k$ be a nonnegative integer. The $k$ th-order jet bundle of $E$, denoted by $J^{k}(E)$, is defined to be the following direct image on $X$ :

$$
J^{k}(E):=p_{1 *}\left(\frac{p_{2}^{*} E}{p_{2}^{*} E \otimes \mathcal{O}_{X \times X}(-(k+1) \Delta)}\right),
$$

where $p_{i}: X \times X \rightarrow X, i=1,2$, is the projection onto the $i$ th factor and $\Delta$ is the diagonal divisor on $X \times X$ consisting of all points of the form $(x, x)$.

Let $K_{X}$ denote the holomorphic cotangent bundle of $X$. There is a natural exact sequence

$$
0 \longrightarrow K_{X}^{\otimes k} \otimes E \longrightarrow J^{k}(E) \longrightarrow J^{k-1}(E) \longrightarrow 0
$$


which is constructed using the obvious inclusion of $O_{X \times X}(-(k+1) \Delta)$ in $0_{X \times X}(-k \Delta)$. The inclusion map $K_{X}^{\otimes k} \otimes E \rightarrow J^{k}(E)$ is constructed by using the homomorphism

$$
K_{X}^{\otimes k} \longrightarrow J^{k}\left(\mathrm{O}_{X}\right)
$$

which is defined at any $x \in X$ by sending $(d f)^{\otimes k}$, where $f$ is any holomorphic function with $f(x)=0$, to the jet of the function $f^{k} / k$ ! at $x$. Any homomorphism $E \rightarrow F$ induces a homomorphism

$$
J^{k}(E) \longrightarrow J^{k}(F)
$$

for any $k \geq 0$.

The sheaf of differential operators $\operatorname{Diff}_{X}^{k}(E, F)$ is defined to be $\operatorname{Hom}\left(J^{k}(E), F\right)$. The homomorphism

$$
\sigma: \operatorname{Diff}_{X}^{k}(E, F) \longrightarrow \operatorname{Hom}\left(K_{X}^{\otimes k} \otimes E, F\right)
$$

obtained by restricting a homomorphism from $J^{k}(E)$ to $F$ to the subsheaf $K_{X}^{\otimes k} \otimes$ $E$ in (2.2), is known as the symbol map.

For all $k, l \geq 0$, there is a natural injective homomorphism

$$
\tau: J^{k+l}(E) \longrightarrow J^{k}\left(J^{l}(E)\right) .
$$

A holomorphic section of $E$ over an open subset $U$ of $X$ gives a section of $J^{i}(E)$ over $U$ for each $i \geq 0$. This defines a map from a subspace of the space of sections $\Gamma\left(U, J^{k+l}(E)\right)$ to a subspace of $\Gamma\left(U, J^{k}\left(J^{l}(E)\right)\right)$. This map extends to the $\mathbb{O}_{X}$-linear homomorphism $\tau$. We describe the image of $\tau$ for the special case $k=1$. This will be done using induction on $l$.

So, first set $l=1$. Using (2.4), the homomorphism $J^{1}(E) \rightarrow E$ in (2.2) gives a homomorphism $\gamma: J^{1}\left(J^{1}(E)\right) \rightarrow J^{1}(E)$. On the other hand, (2.2) gives a homomorphism $\gamma^{\prime}: J^{1}\left(J^{1}(E)\right) \rightarrow J^{1}(E)$. The image $\tau\left(J^{2}(E)\right)$ is the kernel of the difference $\gamma-\gamma^{\prime}$. In other words, we have an exact sequence

$$
0 \longrightarrow J^{2}(E) \stackrel{\tau}{\longrightarrow} J^{1}\left(J^{1}(E)\right) \stackrel{\gamma-\gamma^{\prime}}{\longrightarrow} K_{X} \otimes E \longrightarrow 0 .
$$

Note that the image of $\gamma-\gamma^{\prime}$ is contained in the subbundle $K_{X} \otimes E \subset J^{1}(E)$ since the two projections of $J^{1}\left(J^{1}(E)\right)$ to $E$, obtained from $\gamma$ and $\gamma^{\prime}$, respectively, coincide.

Now, suppose that

$$
\tau_{l-1}: J^{1}(E) \rightarrow J^{1}\left(J^{l-1}(E)\right)
$$

is the homomorphism in (2.6). As before, the projection $J^{l}(E) \rightarrow J^{l-1}(E)$ in (2.2) induces, using (2.4), a homomorphism

$$
\gamma: J^{1}\left(J^{l}(E)\right) \longrightarrow J^{1}\left(J^{l-1}(E)\right) .
$$


On the other hand, consider the homomorphism $J^{1}\left(J^{l}(E)\right) \rightarrow J^{l}(E)$ defined as in (2.2) and let

$$
\gamma^{\prime}: J^{1}\left(J^{l}(E)\right) \longrightarrow J^{1}\left(J^{l-1}(E)\right)
$$

denote its composition with the homomorphism $\tau_{l-1}$ in (2.8). Just as before, the image of the homomorphism

$$
\tau_{l}: J^{1+l}(E) \longrightarrow J^{1}\left(J^{l}(E)\right)
$$

in (2.6) is precisely the kernel of $\gamma-\gamma^{\prime}$.

A holomorphic connection on a holomorphic vector bundle $E$ over $X$ is a first-order operator

$$
\nabla \in H^{0}\left(X, \operatorname{Diff}_{X}^{1}\left(E, K_{X} \otimes E\right)\right)
$$

with the identity automorphism of $E$ as its symbol. It is easy to see that this condition on $E$ is equivalent to the Leibniz identity

$$
\nabla(f s)=d f \otimes s+f \nabla s
$$

where $s$ is a local (holomorphic) section of $E$ and $f$ is a local holomorphic function. The (complex) dimension of $X$ being one, the curvature of $\nabla$ vanishes identically. Therefore, if $\bar{\partial}$ is the Dolbeault operator defining the holomorphic structure of $E$, then $\nabla+\bar{\partial}$ is a flat connection on $E$ compatible with its holomorphic structure. Conversely, the $(1,0)$-part of any flat connection on $E$ compatible with its holomorphic structure defines a holomorphic connection on $E$. By a flat connection on a holomorphic vector bundle we always mean one which is compatible with the holomorphic structure.

For a subbundle $F$ of $E$, the second fundamental form of a holomorphic connection $\nabla$ on $E$ is the composition

$$
F \hookrightarrow E \stackrel{\nabla}{\longrightarrow} K_{X} \otimes E \stackrel{\mathrm{Id} \times q}{\longrightarrow} K_{X} \otimes(E / F),
$$

where the final homomorphism is defined using the natural projection $q: E \rightarrow$ $E / F$. Note that the Leibniz identity ensures that the second fundamental form is a homomorphism of $\mathrm{O}_{X}$-modules. Therefore, it defines a vector bundle homomorphism

$$
S \in H^{0}\left(X, K_{X} \otimes \operatorname{Hom}(F, E / F)\right) .
$$

Now, $S$ generates a subbundle of $E$ containing $F$. Indeed, denoting by $T$ the torsion part of the cokernel $(E / F) / S\left(K_{X}^{*} \otimes F\right)$, for the homomorphism $S$, the inverse image of $T$ for the quotient map $E / F \rightarrow(E / F) / S\left(K_{X}^{*} \otimes F\right)$ is a subbundle of $E / F$ containing the image, under $S$, of $K_{X}^{*} \otimes F$. Denoting this subbundle by 
$F^{\prime}$, the inverse image $F_{2}:=q^{-1}\left(F^{\prime}\right)$ is a subbundle of $E$ containing $F$. Clearly, $F$ is preserved by $\nabla$ if and only if $F=F_{2}$.

We have a filtration

$$
F_{0}:=0 \subset F_{1}:=F \subset F_{2} \subset F_{3} \subset \cdots \subset F_{n} \subset F_{n+1}
$$

of $E$ by subbundles, where $F_{i+1}$ is the subbundle obtained by substituting $F_{i}$ for $F$ in the above construction of $F_{2}$. If $F_{n+1}$, namely where the iterated construction of filtration stabilizes, is a proper subbundle of $E$, then we will define $F_{n+2}$ to be $E$ itself. Note that $F_{n+1}$ is preserved by $\nabla$.

Since the operator $\nabla$ sends $F_{i}$ to $K_{X} \otimes F_{i+1}$, the second fundamental forms for the subbundles in the filtration (2.16) of $E$ give a homomorphism

$$
S_{i}: F_{i} / F_{i-1} \longrightarrow K_{X} \otimes\left(F_{i+1} / F_{i}\right)
$$

of vector bundles for each $i \in[1, n+1]$. So, $S_{1}$ coincides with $S$ in (2.15). Also, if $F_{n+1} \neq E$, then $S_{n+1}=0$ (so, $S_{n+1}$ always vanishes) and $S_{i} \neq 0$ for all $i \leq n$.

3. Coupled connection. Let $X$ be a compact connected Riemann surface of genus at least two. Let $E$ be a holomorphic vector bundle, of rank $(n+1) r$ over $X$, equipped with a holomorphic connection $\nabla$. Suppose $F$ is a holomorphic subbundle of $E$ of rank $r$.

DEFINITION 3.1. The pair $(\nabla, F)$ will be called a coupled connection if $F_{n+1}$, namely where the filtration (2.16) stabilizes, coincides with $E$ and the vector bundle homomorphism $S_{i}$ in (2.17) is an isomorphism for each $i \in[1, n]$. The rank of the subbundle $F$, namely $r$, will be called the rank of the coupled connection.

Take a coupled connection $(\nabla, F)$ on a vector bundle $E$ over $X$.

The final quotient $E / F_{n}$ for the filtration (2.16) of $E$ will be denoted by $Q$. Since each $S_{i}$ in (2.17) is an isomorphism, we have

$$
S_{n} \circ S_{n-1} \circ \cdots \circ S_{1}: F \longrightarrow K_{X}^{n} \otimes Q
$$

as an isomorphism. Here, $S_{i}$ denotes the homomorphism

$$
K_{X}^{i-1} \otimes\left(F_{i} / F_{i-1}\right) \longrightarrow K_{X}^{i-1} \otimes K_{X} \otimes\left(F_{i+1} / F_{i}\right)=K_{X}^{i} \otimes\left(F_{i} / F_{i-1}\right)
$$

obtained by taking the tensor product of the identity map of $K_{X}^{i-1}$ with the homomorphism in (2.17). Furthermore, $F_{i+1} / F_{i}=K_{X}^{\otimes i} \otimes F$.

Let $q: E \rightarrow Q$ denote the natural quotient homomorphism. For any $x \in X$ and any vector $v \in E_{x}$ in the fiber of $E$ over $x$, let $s_{v}$ denote the (unique) local flat section of $E$, defined around $x$, with $s_{v}(x)=v$. Let $\bar{v}$ denote the vector in $J^{j}(Q)$ representing the (local) section $q\left(s_{v}\right)$ of $Q$. Consequently, we have a 
vector bundle homomorphism

$$
\phi_{j}: E \longrightarrow J^{j}(Q)
$$

that sends any $v$ to $\bar{v}$ constructed above.

LEMMA 3.2. The homomorphism $\phi_{n}$ is an isomorphism. Furthermore, there is a commutative diagram

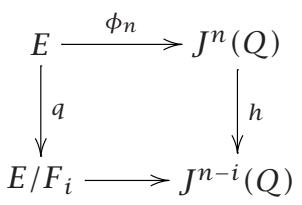

for every $i \in[1, n]$, where $q$ is the natural quotient map and $h$ is obtained from (2.2).

Proof. The lemma follows immediately by unraveling the definitions. Towards this, we first describe the second fundamental form and the filtration in (2.16).

As in (2.15), let $F$ be a subbundle of a flat vector bundle $E$. We give an interpretation of the homomorphism $S$ in (2.15). Take a point $x \in X$ and a vector $v \in F_{x}$ over $x$. Let $v_{1}$ denote the (unique) flat section of $E$ over the first-order infinitesimal neighborhood of $x$, with $v_{1}(x)=v$. Let $\bar{v}$ be a section of $F$ over the first-order infinitesimal neighborhood of $x$, with $\bar{v}(x)=v$. The difference $\bar{v}-v_{1}$ is clearly an element of $\left(K_{X} \otimes E\right)_{x}$. Its projection to $\left(K_{X} \otimes(E / F)\right)_{x}$ coincides with $S(x)(v)$.

More generally, for $i \geq 1$, let $v_{i}$ denote the (unique) flat section of $E$ over the $i$ th-order infinitesimal neighborhood of $x$, with $v_{i}(x)=v$. Let $\bar{v}$ be a section of $F_{i}$ over the $i$ th-order infinitesimal neighborhood of $x$, with $\bar{v}(x)=v$. Consider the difference $\bar{v}_{i}:=\bar{v}-v_{i}$. Using induction, it is easy to see that $v_{i}$ is a section of $F_{i+1}$. Consequently, by projecting $\bar{v}_{i}$, we get an element in $\left(K_{X}^{\otimes i} \otimes\left(F_{i+1} / F_{i}\right)\right)_{x}$. This element in $\left(K_{X}^{\otimes i} \otimes\left(F_{i+1} / F_{i}\right)\right)_{x}$ coincides with $\left(S_{i} \circ S_{i-1} \circ \cdots \circ S_{1}\right)(v)$ with $S_{j}$ as in (3.1). From this observation the lemma follows immediately.

From Lemma 3.2 it follows that we have the homomorphism

$$
\phi_{n+1} \circ \phi_{n}^{-1}: J^{n}(Q) \longrightarrow J^{n+1}(Q)
$$

which is a splitting of the surjective homomorphism $J^{n+1}(Q) \rightarrow J^{n}(Q)$ given by (2.2). In other words, the homomorphism in (3.5) gives a splitting of the exact sequence in (2.2). The resulting homomorphism

$$
\psi: J^{n+1}(Q) \longrightarrow K_{X}^{n+1} \otimes Q
$$


for this splitting is a differential operator

$$
D \in H^{0}\left(X, \operatorname{Diff}_{X}^{n+1}\left(Q, K_{X}^{n+1} \otimes Q\right)\right)
$$

of order $n+1$ on $X$. Since $D$ is defined by a splitting of the jet sequence, its symbol, which is a holomorphic section of $K_{X}^{-n-1} \otimes Q^{*} \otimes K_{X}^{n+1} \otimes Q=\operatorname{End}(Q)$, coincides with the identity automorphism of $Q$.

In Section 4, we see that the above construction of differential operator from a coupled connection can be reversed.

Transport the connection $\nabla$ on $E$ to a connection on $J^{n}(Q)$ using the isomorphism $\phi_{n}$ in (3.3). Consider the filtration (2.16) of $J^{n}(Q)$ for the subbundle $K_{X}^{n} \otimes Q \subset J^{n}(Q)$ in (2.2) corresponding to this connection. Lemma 3.2 says that this filtration of $J^{n}(Q)$ coincides with the filtration obtained from (2.2). Therefore, we have the following equivalent definition of a coupled connection.

DEFINITION 3.3. A coupled connection is a flat connection on $J^{n}(Q)$, where $Q$ is a holomorphic vector bundle on $X$, such that the filtration of $J^{n}(Q)$ obtained from (2.2) coincides with the filtration (2.16) for the subbundle $K_{X}^{n} \otimes Q \subset$ $J^{n}(Q)$.

Note that for the filtration of the vector bundle $J^{n}(Q)$ obtained from (2.2), the graded object is $\oplus_{i=0}^{n} K_{X}^{\otimes i} \otimes Q$. Therefore, if this filtration coincides with the filtration obtained from (2.16), then each homomorphism $S_{i}$ in (2.17) must be an isomorphism. Indeed, this is a consequence of the general fact that any homomorphism between holomorphic vector bundles of the same degree over $X$, which is an isomorphism outside finitely many points, must be an isomorphism.

A holomorphic vector bundle $V$ over $X$ is called stable if for every proper holomorphic subbundle $W \subset V$ the inequality

$$
\operatorname{degree}(W) / \operatorname{rank}(W)<\operatorname{degree}(V) / \operatorname{rank}(V)
$$

is valid $[4,5]$.

DEFINITION 3.4. A coupled connection $(\nabla, F)$ will be called stable if the vector bundle $F$ is stable.

Note that since $K_{X}^{\otimes n} \otimes Q=F$, the vector bundle $Q$ is stable if and only if $F$ is stable.

LEMMA 3.5. Let $Q$ be a stable vector bundle and $\nabla$ a flat connection on $J^{n}(Q)$. Then $\nabla$ is a coupled connection.

Proof. Consider the filtration

$$
0:=V_{0} \subset V_{1}:=K_{X}^{\otimes n} \otimes Q \subset V_{2} \subset V_{3} \subset \cdots \subset V_{n} \subset V_{n+1}=J^{n}(Q)
$$


obtained from the jet sequence (2.2). In other words, $V_{i}$ is the kernel of the projection $J^{n}(Q) \rightarrow J^{n-i}(Q)$ in (2.2). The quotient $V_{i+1} / V_{i}$ is $K_{X}^{n-i} \otimes Q$, which is a stable vector bundle of degree $(g-1)(n-2 i) r$, where $g=\operatorname{genus}(X)$ and $r=\operatorname{rank}(Q)$. The degree is computed from the fact that degree $\left(J^{n}(Q)\right)=0$ since it admits a flat connection. From this, it follows that there is no nonzero homomorphism from $V_{i} / V_{i-1}$ to $K_{X} \otimes\left(V / V_{i+1}\right)$. Indeed, it is easy to see that $H^{0}\left(X, \operatorname{Hom}\left(W_{1}, W_{2}\right)\right)=0$, where $W_{1}$ and $W_{2}$ are stable vector bundles with $\operatorname{degree}\left(W_{1}\right) / \operatorname{rank}\left(W_{1}\right)>\operatorname{degree}\left(W_{2}\right) / \operatorname{rank}\left(W_{2}\right)$. Consequently, if $i \leq n-1$, then

$$
H^{0}\left(X, \operatorname{Hom}\left(V_{i} / V_{i-1}, K_{X} \otimes\left(V_{n+1} / V_{n}\right)\right)\right)=0
$$

as degree $\left(K_{X}\right)>0$. Using the same argument, it also follows that if

$$
H^{0}\left(X, \operatorname{Hom}\left(V_{i} / V_{i-1}, K_{X} \otimes\left(V_{n+1} / V_{j+1}\right)\right)\right)=0,
$$

for some $j \geq i+1$, then $H^{0}\left(X, \operatorname{Hom}\left(V_{i} / V_{i-1}, K_{X} \otimes\left(V_{n+1} / V_{j}\right)\right)\right)=0$. Therefore, using induction, it follows that

$$
H^{0}\left(X, \operatorname{Hom}\left(V_{i} / V_{i-1}, K_{X} \otimes\left(J^{n}(Q) / V_{i+1}\right)\right)\right)=0 .
$$

For a subbundle $W$ of $J^{n}(Q)$, let $S(W): W \rightarrow K_{X} \otimes\left(J^{n}(Q) / W\right)$ denote the second fundamental form of $W$ for the connection $\nabla$. For a subbundle $W_{1}$ of $W$, clearly, there is commutative diagram

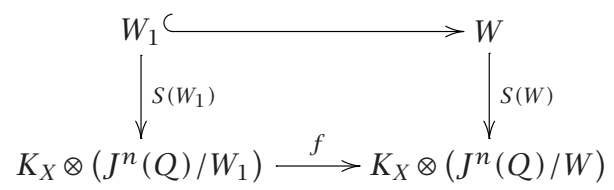

of vector bundles, where $f$ is the tensor product of the identity automorphism of $K_{X}$ with the obvious quotient map $J^{n}(Q) / W_{1} \rightarrow J^{n}(Q) / W$. Using this and (3.12) it follows that

$$
S\left(V_{i}\right) \subset K_{X} \otimes\left(V_{i+1} / V_{i}\right) \subset K_{X} \otimes\left(J^{n}(Q) / V_{i}\right) .
$$

Indeed, (3.14) is immediate for $i=1$. It is straightforward to deduce (3.14) using induction on $i$.

Let

$$
\bar{S}\left(V_{i}\right): V_{i} / V_{i-1} \longrightarrow K_{X} \otimes\left(V_{i+1} / V_{i}\right)
$$

be the homomorphism induced by $S\left(V_{i}\right)$ in (3.14). Since $V_{i} / V_{i-1}$ and $K_{X} \otimes$ $\left(V_{i+1} / V_{i}\right)$ are both stable vector bundles of the same degree/rank quotient, the homomorphism $\bar{S}\left(V_{i}\right)$ must be either an isomorphism or the zero homomorphism. In view of (3.14), it is obvious that if $\bar{S}\left(V_{i}\right)=0$, then $V_{i}$ must be a flat subbundle of $J^{n}(Q)$. But degree $\left(V_{i}\right) \neq 0$ if $i \in[1, n]$, and hence such a 
$V_{i}$ does not admit a flat connection. Therefore, $\bar{S}\left(V_{i}\right)$ is an isomorphism. This completes the proof of the lemma.

The following lemma complements Lemma 3.5.

LEMMA 3.6. Let $Q$ be a stable vector bundle over $X$ of rank $r$ and degree $(1-$ g) $n r$. Then $J^{n}(Q)$ admits a flat connection.

Proof. A theorem of Weil [6] and Atiyah [1] says that a holomorphic vector bundle $V$ over a compact Riemann surface admits a flat connection if and only if every direct summand of it is of degree zero. A holomorphic subbundle $W$ of $V$ is called a direct summand if there is another subbundle $W^{\prime}$ of $V$ such that $W \oplus W^{\prime}$ is isomorphic to $V$. Therefore, every irreducible, that is nondecomposable, holomorphic vector bundle of degree zero admits a flat connection. Consequently, it suffices to show that $J^{n}(Q)$ is irreducible.

Consider the filtration of $J^{n}(Q)$ in (3.9) obtained from jet sequence. We noted that the quotient $V_{i+1} / V_{i}$ is a stable vector bundle of degree $(g-1)(n-$ 2i) $r$. Therefore, the filtration (3.9) is the Harder-Narasimhan filtration of $J^{n}(Q)$. This implies that any automorphism of $J^{n}(Q)$ preserves the filtration (3.9). See [4] for properties of Harder-Narasimhan filtration.

Assume that $J^{n}(Q)=W \oplus W^{\prime}$. Take two nonzero numbers $c, c^{\prime} \in \mathbb{C}^{*}$ with $c \neq c^{\prime}$. Let $T$ denote the automorphism $c \operatorname{Id}_{W} \oplus c^{\prime} \operatorname{Id}_{W^{\prime}}$ of $J^{n}(Q)$ that acts on $W$ as multiplication by $c$ and acts on $W^{\prime}$ as multiplication by $c^{\prime}$.

Since $T$ preserves the filtration (3.9) and $V_{1}$ is simple, we conclude that $V_{1}$ is contained in either $W$ or $W^{\prime}$. Say that $W$ contains $V_{1}$. So $J^{n}(Q) / V_{1}=$ $\left(W / V_{1}\right) \oplus W^{\prime}$, and $T$ gives an automorphism of $J^{n}(Q) / V_{1}$. This automorphism of $J^{n}(Q) / V_{1}$ will be denoted by $T_{1}$.

Since

$$
V_{2} / V_{1} \subset V_{3} / V_{1} \subset \cdots \subset V_{n} / V_{1} \subset V_{n+1} / V_{1}=J^{n}(Q) / V_{1}
$$

is the Harder-Narasimhan filtration of $J^{n}(Q) / V_{1}$, and $V_{2} / V_{1}$ is simple, it follows that $V_{2} / V_{1}$ is contained in either $W / V_{1}$ or $W^{\prime}$. If $V_{2} / V_{1}$ is contained in $W^{\prime}$, then the subbundle $V_{2} \cap W^{\prime}$ of $V_{2}$ gives a splitting of the exact sequence

$$
0 \longrightarrow V_{1} \longrightarrow V_{2} \longrightarrow V_{2} / V_{1} \longrightarrow 0
$$

But each exact sequence

$$
0 \longrightarrow V_{i} / V_{i-1} \longrightarrow V_{i+1} / V_{i-1} \longrightarrow V_{i+1} / V_{i} \longrightarrow 0
$$

is nonsplit, where $i \in[1, n]$. Indeed, the corresponding extension class in

$$
H^{1}\left(X, \operatorname{Hom}\left(V_{i+1} / V_{i}, V_{i} / V_{i-1}\right)\right) \quad\left(\supset H^{1}\left(X, K_{X}\right)\right)
$$

coincides with the element $1 \in H^{1}\left(X, K_{X}\right)$. Recall that $\operatorname{Hom}\left(V_{i+1} / V_{i}, V_{i} / V_{i-1}\right)=$ $K_{X} \otimes \operatorname{End}\left(V_{i+1} / V_{i}\right)$. 
Therefore, we have $V_{2} \subset W$. Now, clearly an inductive argument is valid. More precisely, if $V_{i} \subset W$ and $V_{i+1}$ is not contained in $W$, then $V_{i+1} \cap W^{\prime}$ gives a splitting of the exact sequence (3.18). Using induction, we conclude that $W=$ $V_{n+1}$. In other words, $J^{n}(Q)$ is indecomposable. This completes the proof of the lemma.

Isomorphism classes stable vector bundles of rank $r$ form an algebraic variety of dimension $r^{2}(g-1)+1$ [5]. Consequently, Lemma 3.6 combines with Lemma 3.5 to produce examples of coupled connections.

4. Coupled connections from differential operator. Let $Q$ be a holomorphic vector bundle over $X$ of rank $r$ and let

$$
D \in H^{0}\left(X, \operatorname{Diff}_{X}^{n+1}\left(Q, K_{X}^{n+1} \otimes Q\right)\right)
$$

be a differential operator whose symbol is the identity automorphism of $Q$. The condition on the symbol of $D$ ensures that the homomorphism $J^{n+1}(Q) \rightarrow$ $K_{X}^{n+1} \otimes Q$ defining $D$ gives a splitting of the jet sequence

$$
0 \longrightarrow K_{X}^{n+1} \otimes Q \longrightarrow J^{n+1}(Q) \longrightarrow J^{n}(Q) \longrightarrow 0
$$

Let

$$
\gamma: J^{n}(Q) \longrightarrow J^{n+1}(Q)
$$

be the homomorphism corresponding to this splitting.

Consider the commutative diagram of vector bundles

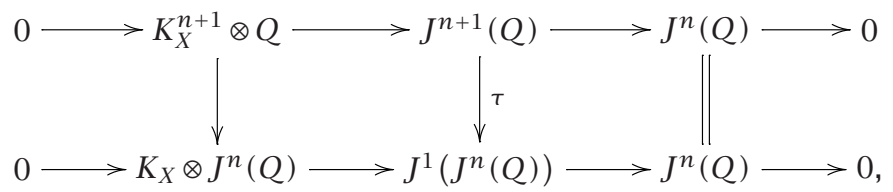

where $\tau$ is defined in (2.6) and the horizontal exact sequences are as in (2.2). The homomorphism

$$
\tau \circ \gamma: J^{n}(Q) \longrightarrow J^{1}\left(J^{n}(Q)\right)
$$

is clearly a splitting of the bottom exact sequence in (4.4), where $\gamma$ is defined in (4.3).

A splitting of the exact sequence

$$
0 \longrightarrow K_{X} \otimes V \longrightarrow J^{1}(V) \longrightarrow V \longrightarrow 0
$$


in (2.2) defines a holomorphic connection on $V$ [1]. More precisely, the space of flat connections on $V$ is in bijective correspondence with the space of splittings of this exact sequence. As we noted earlier, any holomorphic connection on a Riemann surface is automatically flat since there are no nonzero holomorphic 2-forms on it. We recall that given a flat connection on $V$, the corresponding splitting homomorphism $V \rightarrow J^{1}(V)$ of the exact sequence sends any vector $v \in V_{x}$ in the fiber to the image, in $J^{1}(V)$, of the (unique) flat section $s$ of $V$, defined around $x$, with $s(x)=v$.

Therefore, the homomorphism $\tau \circ \gamma$ in (4.5) defines a flat connection on $J^{n}(Q)$. This connection will be denoted by $\bar{\nabla}$.

Given a differential operator $D_{1}$ on $X$ whose symbol homomorphism is an isomorphism, the sheaf of solutions of $D_{1}$ defines a local system on $X$. More precisely, if $D_{1}$ is of order $m$ acting on a vector bundle of rank $l$, then the corresponding local system is of rank $m l$. It can be checked that the local system defined by the operator $D$ in (4.1) coincides with the local system defined by the above flat connection $\bar{\nabla}$.

LEMMA 4.1. The connection $\bar{\nabla}$ on $J^{n}(Q)$ is a coupled connection.

Proof. To prove the lemma, we first give a description of the second fundamental form.

Let $V$ be a vector bundle over $X$ and let $W$ be its subbundle. Consider the commutative diagram

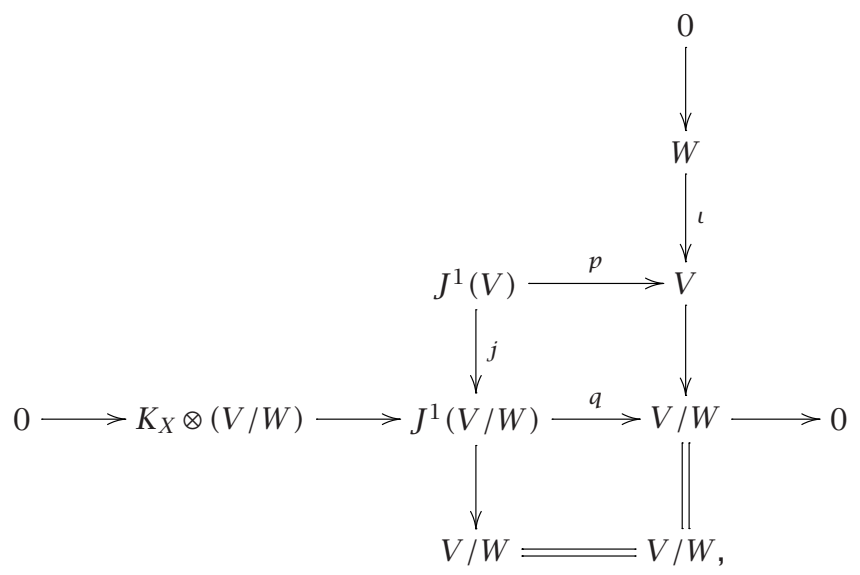

where $\iota$ is the inclusion map and all other maps are as in (2.2). Now let $\nabla$ be a holomorphic connection on $V$. So, $\nabla$ defines a homomorphism

$$
h: V \rightarrow J^{1}(V)
$$

such that $p \circ h$ is the identity automorphism of $V$. 
Consider the homomorphism $j \circ h \circ \iota: W \rightarrow J^{1}(V / W)$. The commutativity of the diagram implies that $q \circ j \circ h \circ \iota=0$. Consequently, $j \circ h \circ \iota$ gives a homomorphism

$$
S(W): W \longrightarrow K_{X} \otimes(V / W)
$$

It is easy to check that this homomorphism coincides with the second fundamental form of $W$ for the connection $\nabla$.

For any $i \in[0, n-1]$, let $W_{i}$ denote the kernel of the homomorphism $J^{n}(Q) \rightarrow$ $J^{i}(Q)$ in (2.2). We have a commutative diagram

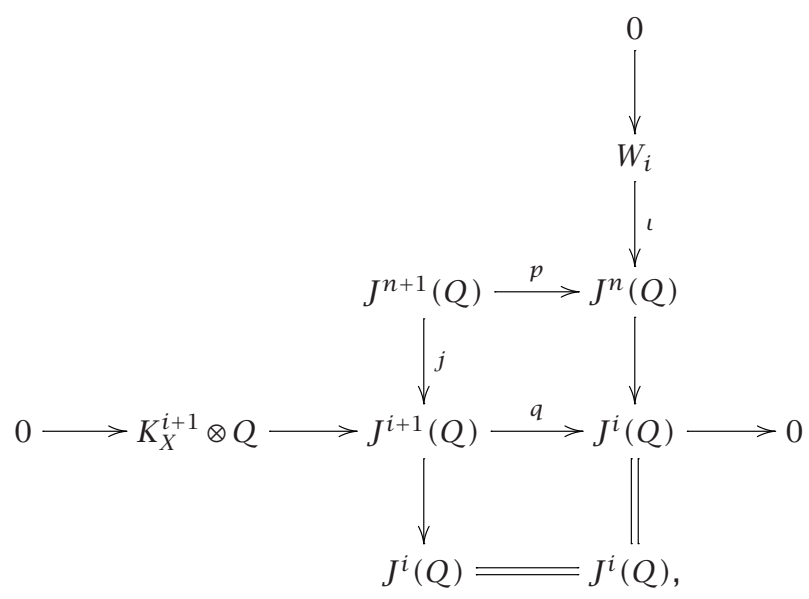

where $\iota$ is the inclusion map and all other maps are as in (2.2). Let $h: J^{n}(Q) \rightarrow$ $J^{n+1}(Q)$ be a splitting of $p$, that is, $p \circ h$ is the identity automorphism of $J^{n}(Q)$. Just as before, the homomorphism $j \circ h \circ \iota$ gives a homomorphism

$$
S_{i}: W_{i} \longrightarrow K_{X}^{i+1} \otimes Q
$$

since $q \circ j \circ h \circ \iota=0$. On the other hand, we have a commutative diagram

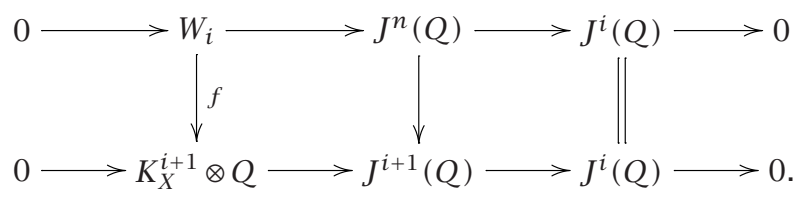

It is straightforward to check that the above homomorphism $f$ coincides with $S_{i}$ constructed in (4.11).

Since the connection $\bar{\nabla}$ is defined by $\tau \circ \gamma$, where $\gamma$ is the splitting of the homomorphism $p$ in (4.10), from the earlier description of the second fundamental form it follows that the second fundamental form of the subbundle $W_{i} \subset J^{n}(Q)$, for the connection $\bar{\nabla}$, coincides with the homomorphism $S_{i}$ in 
(4.11) constructed by substituting $h$ with $\gamma$. But $S_{i}$ coincides with $f$. Therefore, the filtration (2.16) constructed from the subbundle $W_{n-1}=K_{X}^{n} \otimes Q \subset J^{n}(Q)$ for the connection $\bar{\nabla}$ coincides with the filtration (3.9) constructed from (2.2). This completes the proof of the lemma.

In Section 3, given a coupled connection, we constructed a differential operator (see (3.7)). Let

$$
\bar{D} \in H^{0}\left(X, \operatorname{Diff}_{X}^{n+1}\left(Q, K_{X}^{n+1} \otimes Q\right)\right)
$$

be the differential operator for the coupled connection $\left(J^{n}(Q), \bar{\nabla}\right)$ constructed in Lemma 4.1 from the operator $D$ in (4.1).

Proposition 4.2. The differential operator $\bar{D}$ coincides with the operator $D$ in (4.1).

Proof. It is straightforward to check that the homomorphism $\gamma$ in (4.3) coincides with the homomorphism $\phi_{n+1} \circ \phi_{n}^{-1}$ in (3.5) for the coupled connection $\left(J^{n}(Q), \bar{\nabla}\right)$. The proposition follows immediately from this observation.

Let

$$
D^{\prime} \in H^{0}\left(X, \operatorname{Diff}_{X}^{n+1}\left(Q^{\prime}, K_{X}^{n+1} \otimes Q^{\prime}\right)\right)
$$

be a differential operator with the identity automorphism of $Q^{\prime}$ as its symbol (the same condition for $D$ in (4.1)). The two operators $D$ and $D^{\prime}$ will be called equivalent if there is an isomorphism

$$
\rho: Q \longrightarrow Q^{\prime}
$$

which intertwines $D$ and $D^{\prime}$. In other words, $(\operatorname{Id} \otimes \rho) \circ D=D^{\prime} \circ \rho$, where $\operatorname{Id} \otimes \rho$ is the automorphism of $K_{X}^{n+1} \otimes Q$ defined by $\rho$ and the identity automorphism of $K_{X}^{n+1}$.

Let $\mathscr{B}(r, n)$ denote the space of all equivalence classes of differential operators

$$
D \in H^{0}\left(X, \operatorname{Diff}_{X}^{n+1}\left(Q, K_{X}^{n+1} \otimes Q\right)\right)
$$

where $Q$ is some holomorphic vector bundle of rank $r$ and the symbol of $D$ is the identity automorphism of $Q$.

If $D_{1} \in H^{0}\left(X, \operatorname{Diff}_{X}^{n+1}\left(Q, Q_{1}\right)\right)$ is a differential operator whose symbol is an isomorphism, then $Q_{1}$ is isomorphic to $K_{X}^{n+1} \otimes Q$. Therefore, $\mathscr{B}(r, n)$ coincides with the space of equivalence classes of differential operators of order $n+1$, between two vector bundles of rank $r$, such that the symbol is an isomorphism.

If $D$ and $D^{\prime}$ are two equivalent differential operators from $Q$ to $K_{X}^{n+1} \otimes Q$, then the automorphism $\rho$ of $Q$ that intertwines $D$ and $D^{\prime}$ induces an automorphism of $J^{n}(Q)$. Let $\rho^{\prime}$ denote the automorphism of $J^{n}(Q)$. Let $\bar{\nabla}$ (resp., $\bar{\nabla}^{\prime}$ ) 
be the coupled connection constructed from $D$ (resp., $D^{\prime}$ ) in Lemma 4.1. It is easy to see that the automorphism $\rho^{\prime}$ commutes with the connections $\bar{\nabla}$ and $\bar{\nabla}^{\prime}$.

Let $E$ and $E^{\prime}$ be two holomorphic vector bundles over $X$ of rank $(n+1) r$. Let $(\nabla, F)$ and $\left(\nabla^{\prime}, F^{\prime}\right)$ be two coupled connections of rank $r$ on $E$ and $E^{\prime}$, respectively. The two coupled connections will be called equivalent if there is an automorphism

$$
T: E \longrightarrow E^{\prime}
$$

such that $T(F)=F^{\prime}$ and $T$ takes $\nabla$ to $\nabla^{\prime}$, that is, $\nabla^{\prime} \circ T=T \circ \nabla$.

Since $T$ takes $F$ to $F^{\prime}$ and $\nabla$ to $\nabla^{\prime}$, it follows immediately that $T$ takes the filtration (2.16) for $F$ to the corresponding filtration for $F^{\prime}$. In particular, $T$ gives an isomorphism of the final quotient $Q=E / F_{n}$ with the corresponding quotient of $E^{\prime}$.

Let $A(r, n)$ denote the space of all equivalence classes of coupled connections of rank $r$ on some holomorphic vector bundle of rank $(n+1) r$ over $X$.

From the above comment on the construction in Lemma 4.1 it follows that we have a map

$$
\Phi: \mathscr{B}(r, n) \longrightarrow \mathscr{A}(r, n)
$$

that sends an operator $D$ to the coupled connection $\bar{\nabla}$ constructed in Lemma 4.1 from $D$.

Let the two coupled connections $(\nabla, F)$ on $E$ and $\left(\nabla^{\prime}, F^{\prime}\right)$ on $E^{\prime}$, as above, be equivalent. As before, $Q$ and $Q^{\prime}$ denote the final quotient $F_{n+1} / F_{n}$ for $(\nabla, F)$ and $\left(\nabla^{\prime}, F^{\prime}\right)$, respectively. Consider the isomorphism

$$
T^{\prime}: Q \longrightarrow Q^{\prime}
$$

induced by $T$ in (4.17). Let

$$
T_{j}: J^{j}(Q) \longrightarrow J^{j}\left(Q^{\prime}\right)
$$

denote the isomorphism induced by $T^{\prime}$. The diagram

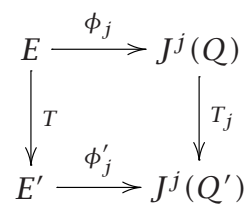

is commutative, where $\phi_{j}$ and $\phi_{j}^{\prime}$ are constructed in (3.3).

From the commutativity of (4.21) it is immediate that the differential operators $D$ and $D^{\prime}$ constructed, as in $(3.7)$, from $(\nabla, F)$ and $\left(\nabla^{\prime}, F^{\prime}\right)$, respectively, are equivalent. More precisely, the isomorphism $T^{\prime}$ takes $D$ to $D^{\prime}$. 
Therefore, we have a map

$$
\Psi: \mathscr{A}(r, n) \longrightarrow \mathscr{B}(r, n)
$$

that sends a coupled connection $(\nabla, F)$ to the differential operator $D$ constructed in (3.7).

We now have the following corollary of Proposition 4.2.

COROLLARY 4.3. The composition $\Psi \circ \Phi$ is the identity map of $\mathscr{B}(r, n)$, where $\Phi$ and $\Psi$ are constructed in (4.18) and (4.22), respectively. In particular, $\Psi$ is surjective and $\Phi$ is injective.

The following lemma complements Corollary 4.3.

LEMMA 4.4. The composition $\Phi \circ \Psi$ is the identity map of $\mathscr{A}(r, n)$.

Proof. Let $\alpha:=(\nabla, F)$ be a coupled connection on $E$ and $\beta:=\left(\nabla^{\prime}, F^{\prime}\right)$ a coupled connection on $E^{\prime}$ such that the differential operator $\Psi(\alpha)$ is equivalent to $\Psi(\beta)$.

Let $Q$ (resp., $Q^{\prime}$ ) denote the final quotient $F_{n+1} / F_{n}$ in (2.16) for $\alpha$ (resp., $\beta$ ). Since $\Psi(\alpha)$ and $\Psi(\beta)$ are equivalent, there is an isomorphism

$$
A: Q \longrightarrow Q^{\prime}
$$

that takes $\Psi(\alpha)$ to $\Psi(\beta)$. In other words, $(\operatorname{Id} \otimes A) \circ \Psi(\alpha)=\Psi(\beta) \circ A$, where Id denotes the identity automorphism of $K_{X}^{n+1}$. Let

$$
\bar{A}: J^{n}(Q) \longrightarrow J^{n}\left(Q^{\prime}\right)
$$

be the isomorphism induced by $A$.

Let $\phi_{n}$ (resp., $\phi_{n}^{\prime}$ ) denote the isomorphism from $E$ (resp., $E^{\prime}$ ) to $J^{n}(Q)$ (resp., $\left.J^{n}\left(Q^{\prime}\right)\right)$ constructed in Lemma 3.2. Finally, consider the isomorphism

$$
T:=\left(\phi_{n}^{\prime}\right)^{-1} \circ \bar{A} \circ \phi_{n}: E \longrightarrow E^{\prime}
$$

The isomorphism $\phi_{n}$ takes the filtration on $E$ obtained from (2.16) to the filtration (3.9) of $J^{n}(Q)$ and the same for $\phi_{n}^{\prime}$. Furthermore, $\bar{A}$ evidently takes the filtration (3.9) of $J^{n}(Q)$ to the corresponding filtration of $J^{n}\left(Q^{\prime}\right)$. Therefore, the isomorphism $T$ in (4.25) is compatible with the filtrations. It is easy to check that $T$ takes the connection $\nabla$ to the connection $\nabla^{\prime}$. This completes the proof of the lemma.

Corollary 4.3 and Lemma 4.4 combine together to give the following theorem.

THEOREM 4.5. The two maps $\Phi$ and $\Psi$ are bijective. Moreover, they are inverses of each other. 
Let $\mathscr{A}^{\prime}(r, n) \subset \mathscr{A}(r, n)$ be the subset defined by stable coupled connections (see Definition 3.4). Similarly, let $\mathscr{B}^{\prime}(r, n) \subset \mathscr{B}(r, n)$ denote the subset consisting of all operators defined on a stable vector bundle.

Theorem 4.5 has the following corollary.

COROLlaRY 4.6. Each of the two maps $\Phi$ and $\Psi$ defines a bijection between $\mathscr{A}^{\prime}(r, n)$ and $\mathscr{S}^{\prime}(r, n)$. Furthermore, they are inverses of each other.

\section{REFERENCES}

[1] M. F. Atiyah, Complex analytic connections in fibre bundles, Trans. Amer. Math. Soc. 85 (1957), 181-207.

[2] I. Biswas, Differential operators and immersions of a Riemann surface into a Grassmannian, J. Geom. Phys. 41 (2002), no. 4, 286-295.

[3] Coupled connections on a compact Riemann surface, J. Math. Pures Appl. (9) 82 (2003), no. 1, 1-42.

[4] S. Kobayashi, Differential Geometry of Complex Vector Bundles, Publications of the Mathematical Society of Japan, vol. 15, Princeton University Press, New Jersey, 1987.

[5] C. S. Seshadri, Fibrés Vectoriels sur les Courbes Algébriques [Vector Bundles over Algebraic Curves], Astérisque, vol. 96, Société Mathématique de France, Paris, 1982 (French).

[6] A. Weil, Généralisation des fonctions abéliennes, J. Math. Pures Appl. 17 (1938), 47-87 (French).

Indranil Biswas: School of Mathematics, Tata Institute of Fundamental Research, Bombay 400005, India

E-mail address: indrani1@math.tifr.res.in 


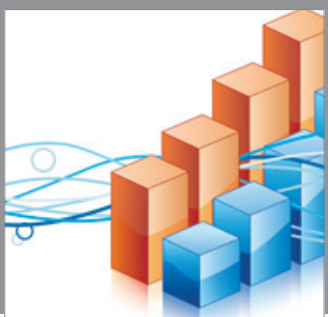

Advances in

Operations Research

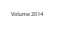

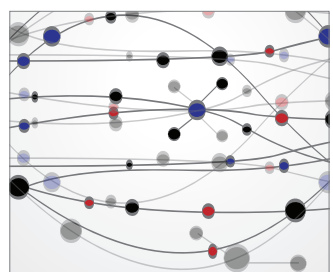

\section{The Scientific} World Journal
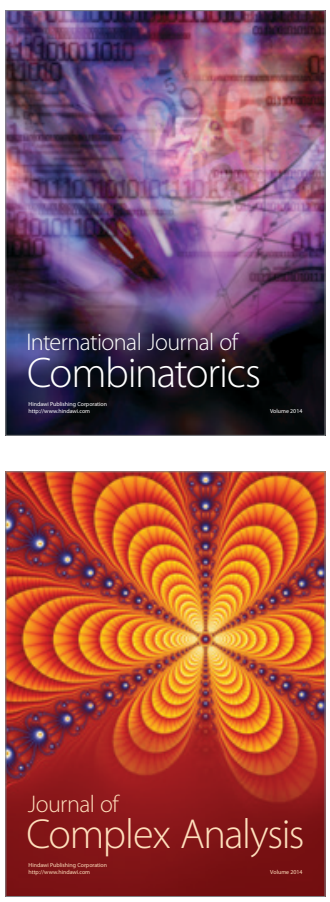

International Journal of

Mathematics and

Mathematical

Sciences
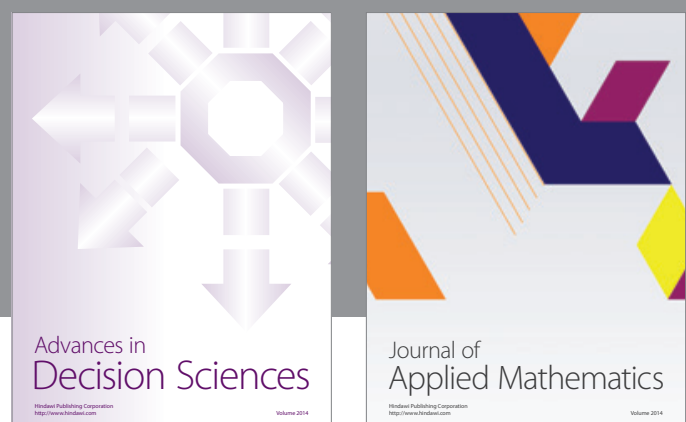

Journal of

Applied Mathematics
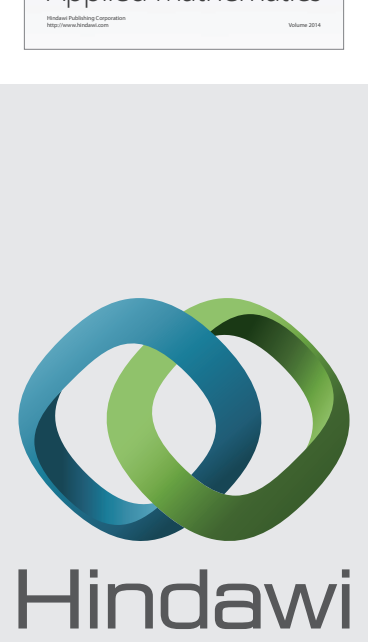

Submit your manuscripts at http://www.hindawi.com
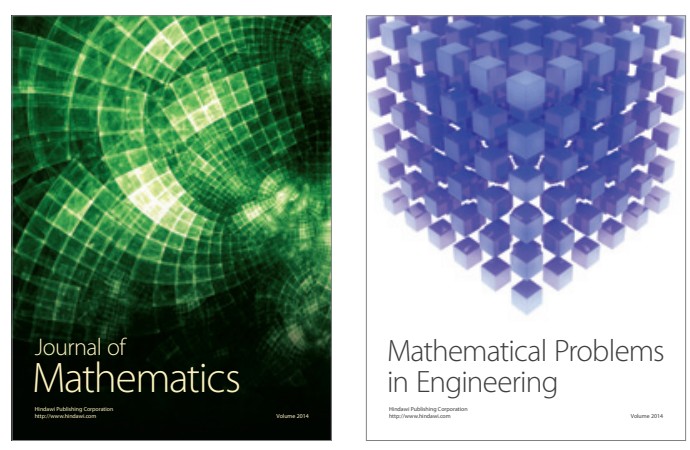

Mathematical Problems in Engineering
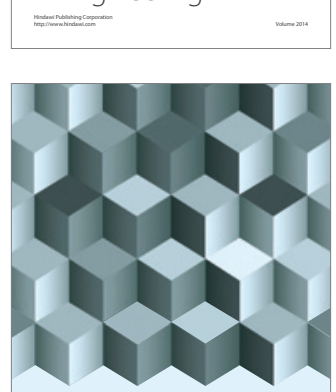

Journal of

Function Spaces
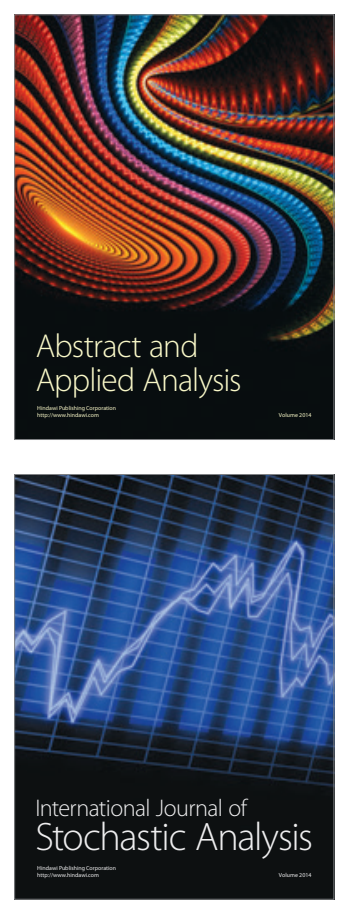

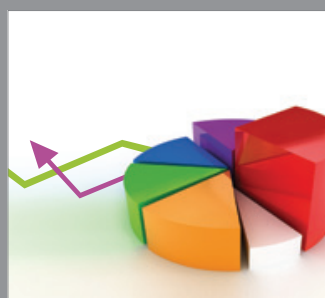

ournal of

Probability and Statistics

Promensencen
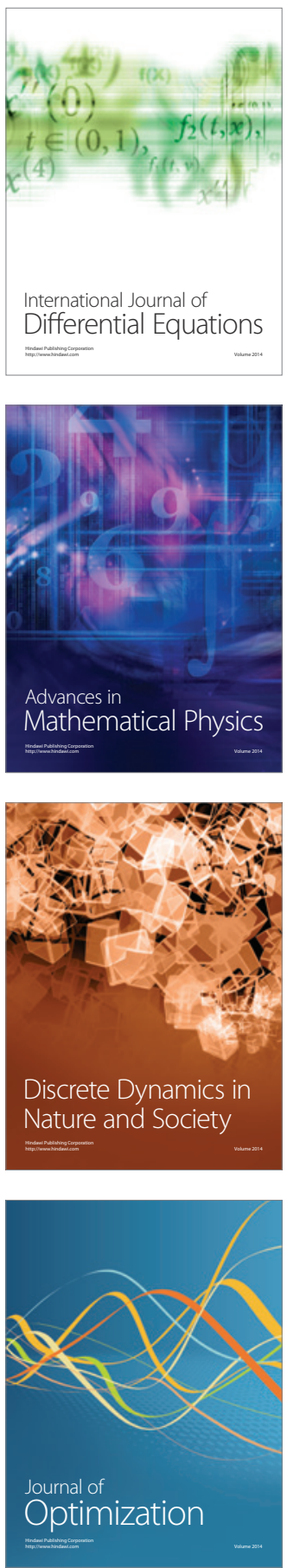